\title{
Stability of Dye-Sensitized Solar Cells under Extended Thermal Stress
}

\author{
Surendra K. Yadav ${ }^{I}$, Sandheep Ravishankar ${ }^{2}$, Sara Pescetelli, Antonio Agresti ${ }^{l}$, Franciso \\ Fabregat-Santiago $^{2}$, Aldo Di Carlo $^{1 *}$
}

1. C.H.O.S.E. (Centre for Hybrid and Organic Solar Energy), Department of Electronic Engineering, University of Rome "Tor Vergata", via del Politecnico 1, Rome, 00133 Italy.

2. Photovoltaics and Optoelectronic Devices Group, Institute of Advanced Materials, Universitat Jaume I, Castello de la Plana, 12006, Spain

\begin{abstract}
Stability of dye-sensitized solar cells (DSCs) is a key issue and of great interest, since it could be a potential candidate for low cost energy production in order to satisfy the global energy demand. This work provides insight on stability of DSCs, assessed through accelerated aging test at $85^{\circ} \mathrm{C}$, followed by Raman spectroscopy and impedance spectroscopy (IS) measurements. The results from DSCs based on two ruthenium-based dyes Z907, Ru505 and two commercially available electrolytes such as high stability electrolyte (HSE) and solvent-free livion 12 (L-12). The least stable device composed of Z907/HSE retains 30\% of its initial efficiency after 4700 hours of thermal stress at $85^{\circ} \mathrm{C}$, while the remarkably stable cells $\mathrm{Ru} 505 / \mathrm{L}-12$ retains $96 \%$ of its initial efficiency with degradation rate of $\sim 14 \% / 1000 \mathrm{~h}$ and $\sim 1 \% / 1000 \mathrm{~h}$ respectively. The present findings show a solution to the degradation problem far beyond standard requirements and could generate strong interest in the photovoltaic industry.
\end{abstract}

Key Words: DSCs, Stability, Aging, hermetic Sealing, Ionic Liquid

\section{Introduction}

The stability of DSCs [1] is an important aspect needing to be addressed prior to their commercialization. However, the evaluation of the solar cells lifetime is a difficult task since it depends on the specific degradation mechanisms [2]. In order to get higher stability, the understanding of degradation mechanisms intrinsic or extrinsic is essential as it will help to develop better materials and fabrication processes to obtain stable solar cells. In general, hermitic sealing of DSCs is essential for long-term operation of the DSCs. The sealing should be mechanically stable to avoid any cell damage due to external factors such as humidity. Moreover, the sealing has to be flexible enough so that it does not break in case of thermal expansion and upsurge of vapor pressure of the electrolyte. [3-5] At the same time, it is required to know which are the intrinsic degradation mechanisms involving the constituent chemical compounds. Several studies have evaluated the intrinsic deterioration under different kinds of aging tests such as reverse bias, thermal stress, temperature cycles, light soaking etc. [6-9]. Some of these harsh stress conditions may be prevented by an adequate protection strategy; for example, reverse bias degradation may be avoided by an effective diode protection retaining low 
voltages across the reverse biased cells. However, in real working conditions, DSCs can undergo severe unavoidable thermal stress (operative temperature up to $80^{\circ} \mathrm{C}$ ) inducing permanent electrolyte degradation and eventually loss of photovoltaic performance. As a matter of fact, the use of volatile solvents in the electrolyte solution together with non-robust sealing frequently results in solvent evaporation and solution leakage with a consequent chemical degradation of the dye molecules till the device completely breaks down. [10]

The recent reports in literature on thermal stability of DSCs was explicitly shown by various researchers, such as R. Harikisun et. al., who reported robust DSC structure showing remarkable thermal stability $\left(1000 \mathrm{hrs}\right.$ in ambient condition at $80^{\circ} \mathrm{C}$ ) by retaining more than $80 \%$ of initial performance [11]. Similarly, T. Stergiopoulos et. al declared DSC long-term stability of about $5000 \mathrm{hrs}$ when non-nitrile solvent for electrolyte solution is employed [12]. In particular, $3000 \mathrm{~h}$ light soaking exposure followed by $2000 \mathrm{~h}$ at $85^{\circ} \mathrm{C}$ induced power conversion efficiency (PCE) reduction of only $10 \%$ in the case of $\gamma$-butyrolactone-based electrolyte $[12,13]$. Thermal aging has also been carried out at $80^{\circ} \mathrm{C}$ for $2000 \mathrm{hrs}$ using tetraglyme high boiling point solvent electrolyte with $20 \%$ loss in efficiency [14]. Moreover, W. Yang and co-workers recently reported DSCs employing a novel cobalt bipyridyl-based electrolyte that pass $1200 \mathrm{~h}$ at $70^{\circ} \mathrm{C}$ test by retaining $80 \%$ of its initial open circuit voltage $\left(V_{o c}\right)$ [15]. In order to evaluate the thermal degradation mechanisms in DSCs, we have used devices employing two different ruthenium based dyes, namely (cis-RuLL' $(\mathrm{SCN})_{2}\left(\mathrm{~L}=4\right.$, 4'-dicarboxylic acid-2,2'-bipyridine, $\mathrm{L}^{\prime}=4,4^{\prime}$ dinonyl-2,2'-bipyridine) Z907, cis-dicyano-bis (2,2-bipyridyl 4,4-dicarboxylic acid) ruthenium(II) (Ru505, Orange Dye) and two different commercially available electrolytes having different viscosity properties, namely High Stability Electrolyte (HSE, Dyesol) based on 3methoxypropionitrile --MPN-- solvent and livion 12, (L-12, Merck) as solvent-free electrolyte (1-methyl-3-propylimidazolium iodide --PMIM-I-- + 1-Ethyl-3-methylimidazolium tetracyanoborate --EMIM-TCB--) in volume ratio of 65/35 respectively. The employed dye molecule structures differ mainly in cyanide and thiocyanate ancillary ligands while the electrolytes present different viscosity and boiling points. The investigated devices are indicated as Z907/HSE, Z907/L-12, Ru505/HSE and Ru505/L-12 by distinguishing them on the basis of dye and electrolyte. Here, we forced accelerated aging of DSCs via thermal stress, coupled with IS measurements to unveil degradation mechanisms under real working conditions. It has been observed that under prolonged thermal stress at $85^{\circ} \mathrm{C}$, severe performance degradation occurred in the devices containing HSE while very promising results are found for devices fabricated with L-12 electrolyte. The results for L-12 are thus promising in terms of stability to qualify the IEC tests protocols. Thus, the use of a solvent-free electrolyte combined with a hermetic sealing strategy can effectively extend the device's lifetime and eventually promote the DSC commercialization.

\section{Experimental Section}


A fluorine-doped tin oxide (FTO, NSG TEC $8 \Omega / \square$ ) conducting glass washed with acetone first then with ethanol and isopropanol at the end according to cleaning protocol. The mesoporous transparent film of $\mathrm{TiO}_{2}$ (transparent $\mathrm{TiO}_{2}$ paste DSL 18NR-T, Dyesol) was deposited on to the pre-cleaned FTO glass plate by screen printing. The $\mathrm{TiO}_{2}$ film was dried at $\sim 80{ }^{\circ} \mathrm{C}$ for 20 min and then fired at $450{ }^{\circ} \mathrm{C}$ for $\sim 30$ min to remove organic materials and to form crystalline $\mathrm{TiO}_{2}$ mesoporous film structure. $\mathrm{TiO}_{2}$ electrode was gradually sintered in a furnace under an air flow with a temperature ramp rate of $15{ }^{\circ} \mathrm{C} / \mathrm{min}$. The final thickness of the $\mathrm{TiO}_{2}$ film after sintering was $7 \mu \mathrm{m}$ as measured by Profilometer (Dektak 150, Veeco). When the temperature was cooled down to $80^{\circ} \mathrm{C}$, the $\mathrm{TiO}_{2}$ electrodes were immersed into a $\mathrm{Z907}$ (Dyesol) ruthenium dye (cis$\mathrm{RuLL}^{\prime}(\mathrm{SCN})_{2}$ ( $\mathrm{L}=4,4^{\prime}$-dicarboxylic acid-2,2'-bipyridine, $\mathrm{L}^{\prime}=4,4^{\prime}$-dinonyl-2,2'-bipyridine) or cis-dicyano-bis (2,2-bipyridyl 4,4-dicarboxylic acid) ruthenium(II) (Ru505) solutions in ethanol and isopropanol respectively for 16 hours in the dark to absorb the dye onto $\mathrm{TiO}_{2}$ surface, in this way we obtain the Photoelectrode (PE). The counter electrode (CE) was prepared by screenprinting a platinum paste (Platinum Paste, Dyesol Ltd.) on a FTO/glass substrate, followed by firing at $450^{\circ} \mathrm{C}$ for $30 \mathrm{~min}$ reached with a temperature ramp rate of $15{ }^{\circ} \mathrm{C} / \mathrm{min}$. Screen printed silver paste (Dupont) strips were used to make contacts between cells and external circuit output. Silver paste was fired at $450^{\circ} \mathrm{C}$ for $30 \mathrm{~min}$. DSCs were assembled by sealing the CE and the dyecoated PE with a heat treated before sealing for excellent adhesion, thermo plastic Sealant (40 $\mu \mathrm{m}$ Binel gasket, DuPont) at $150^{\circ} \mathrm{C}$ for $30 \mathrm{~s}$ using hot press equivalent to 1 bar. Electrolyte solutions were injected into the cells, via vacuum back filling technique, through a channel made in the sealant gasket. After electrolyte injection, the channel was closed by hot pressing the channel part of the cell. The active area of the DSC was $(0.9 \mathrm{cmx} 4 \mathrm{~cm}) 3.6 \mathrm{~cm}^{2}$. Each masterplate contains 5 equal cells with an area of $3.6 \mathrm{~cm}^{2}(0.9 \mathrm{~cm} \times 4 \mathrm{~cm})$ each. The area of the complete 1 cell was $(1 \mathrm{cmx} 4.6 \mathrm{~cm}) 4.6 \mathrm{~cm}^{2}$. The additional area in the cells for electrolyte window to monitoring the degradation of electrolyte and dye desorption separately.

In order to evaluate the degradation of solar cells, the cells were subjected to thermal stress at $85^{\circ} \mathrm{C}$. Current density-voltage $(J-V)$ curves recorded using a solar simulator with halogen lamps providing 1 sun illumination $\left(1000 \mathrm{~W} / \mathrm{m}^{2}\right)$ at different stages during stress duration. To estimate aging effects due to temperature stress, the electrical parameters of solar cells such as efficiency $(\eta)$, open circuit voltage $\left(V_{o c}\right)$, short circuit current $\left(J_{s c}\right)$ and fill factor $(F F)$ were monitored. In this work, Micro-Raman spectroscopy was exploited as a site-specific spectroscopic probe to evaluate the structural changes due to degradation in DSCs under prolonged thermal stresses. Micro-Raman measurements were performed by focusing a laser beam of applicable wavelength on $\mathrm{TiO}_{2} /$ Dye photoelectrodes as well as the electrolyte for fresh cells and aged cells. The confocal micro-Raman spectroscopy was employed as a site-specific spectroscopic probe to evaluate the stress induced structural changes for each constituting layer. Raman spectroscopy was conducted through Jobin-Yvon-Horiba micro-Raman system (LabRAM ARAMIS) equipped with $\mathrm{Ar}+$ ion laser $(514 \mathrm{~nm})$ as excitation source $(100 \mathrm{~mW})$. The Horiba micro-spectrometer is coupled with a confocal microscope that allows the spatial resolution of the sample. The spectrometer was equipped with a diffraction grating of 1800 lines $/ \mathrm{mm}$. The laser light reached 
the sample surface at normal incidence by means of ultra-long working distance (50X) objective. The laser spot has a power density of $0.25 \mathrm{~mW} \cdot \mu \mathrm{m}^{-2}$ on the investigated sample. Additionally, IS measurements and dark $J-V$ measurements were carried out using a potentiostat unit by Autolab. The IS analyzed frequency range being from $20 \mathrm{mHz}$ to $100 \mathrm{kHz}$. The EIS measurements were performed in dark conditions over the voltage range of -0.8 to $0 \mathrm{~V}$ in $50-100 \mathrm{mV}$ step intervals. Z-View software from Scribner Inc. was used for fitting the data.

\section{Results and Discussion}

The $J-V$ curves under 1 sun illumination for fresh Z907/HSE and Z907/L-12 cells, show in Fig. 1a). The cells Z907/HSE, Ru505/HSE were more efficient approximately 1.7 and 2.1 times than Z907/L-12 and Ru505/L-12 respectively. The extended absorption for Z907 dye towards higher
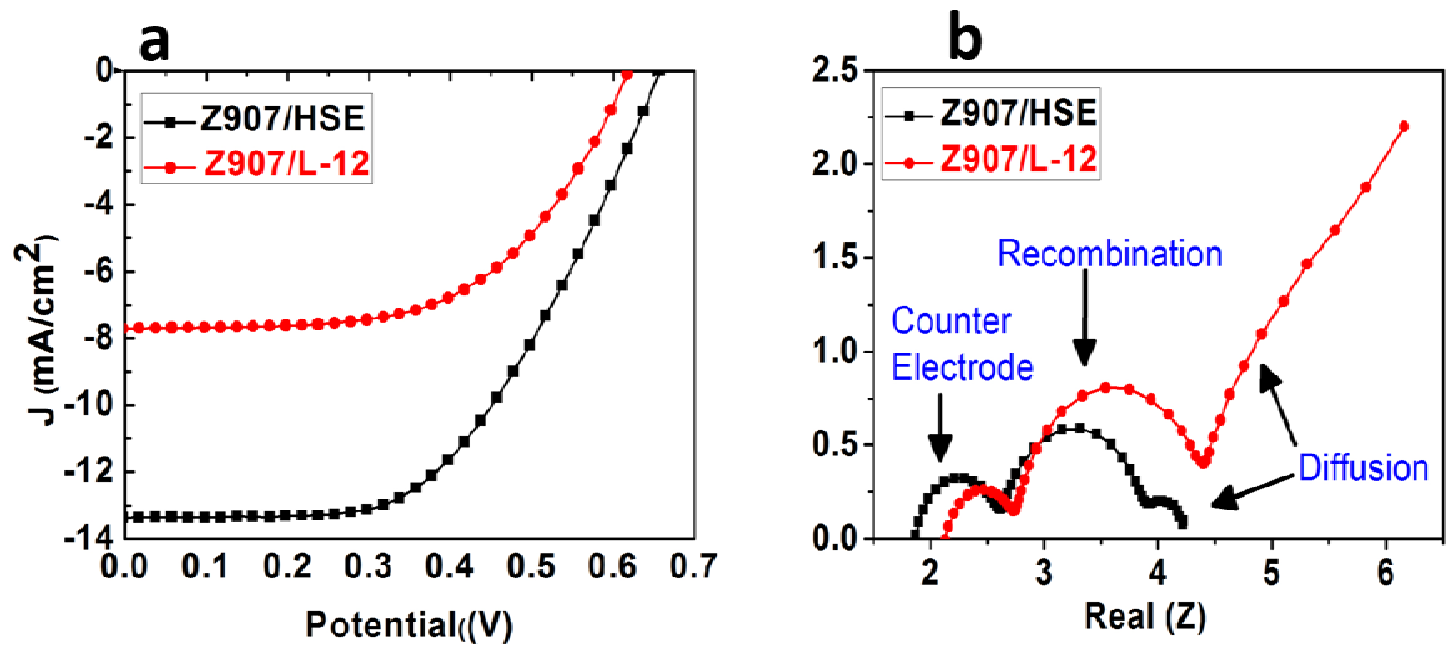

Figure 1 (a) J-V curve at 1 sun illumination for reference cells; Z907/HSE and Z907/L-12. (b) IS spectra of these samples at $\mathrm{V}=0.8 \mathrm{~V}$, recorded in dark.

wavelengths compared to Ru505 reported in Supplementary Information (S1) is the cause of the higher $J_{s c}$ and thus efficiency, observed for devices using Z907 dye as sensitizer. $J_{s c}$ and $V_{o c}$ were both higher in Z907/HSE, Ru505/HSE as compared to the Z907/L-12, Ru505/L-12. The J-V curve for Ru505/HSE and Ru505/L-12 is given in Supplementary Information (S2). Fig. 2 summarizes evolution of four types of samples after 4700 hours of thermal stress at $85^{\circ} \mathrm{C}$. Z907/HSE, Z907/L-12, Ru505/HSE and Ru505/L-12 devices retain 30\%, 60\%, 65\% and 95\% of their initial efficiency respectively. Thus, devices with L-12 electrolyte and Ru505 dye are the ones presenting slower degradation rate under thermal stress. In particular, device Ru505/L-12 shows the slowest degradation rate reflected as $4 \%, 3 \%$ and $7 \%$ loss in efficiency, $j_{s c}$ and $V_{o c}$ respectively.

Several mechanisms are observed that affect the degradation from the least stable Z907/HSE to the most stable Ru505/L-12. In case of HSE-based devices, the first performance reduction is associated with electrolyte leakage during 4700 hours of thermal stress. While devices 
employing L-12 electrolyte show no leakage. The devices employing HSE degradation effect produces the large current losses occurring in aged samples and is responsible of $50 \%$ to $80 \%$ performance loss in the cells with Ru505 and Z907 respectively. HSE has a larger thermal expansion coefficient than that of L-12 and for this reason, the stress induced by the thermal expansion at high temperature of HSE on the sealant is larger than the one induced by L-12. As
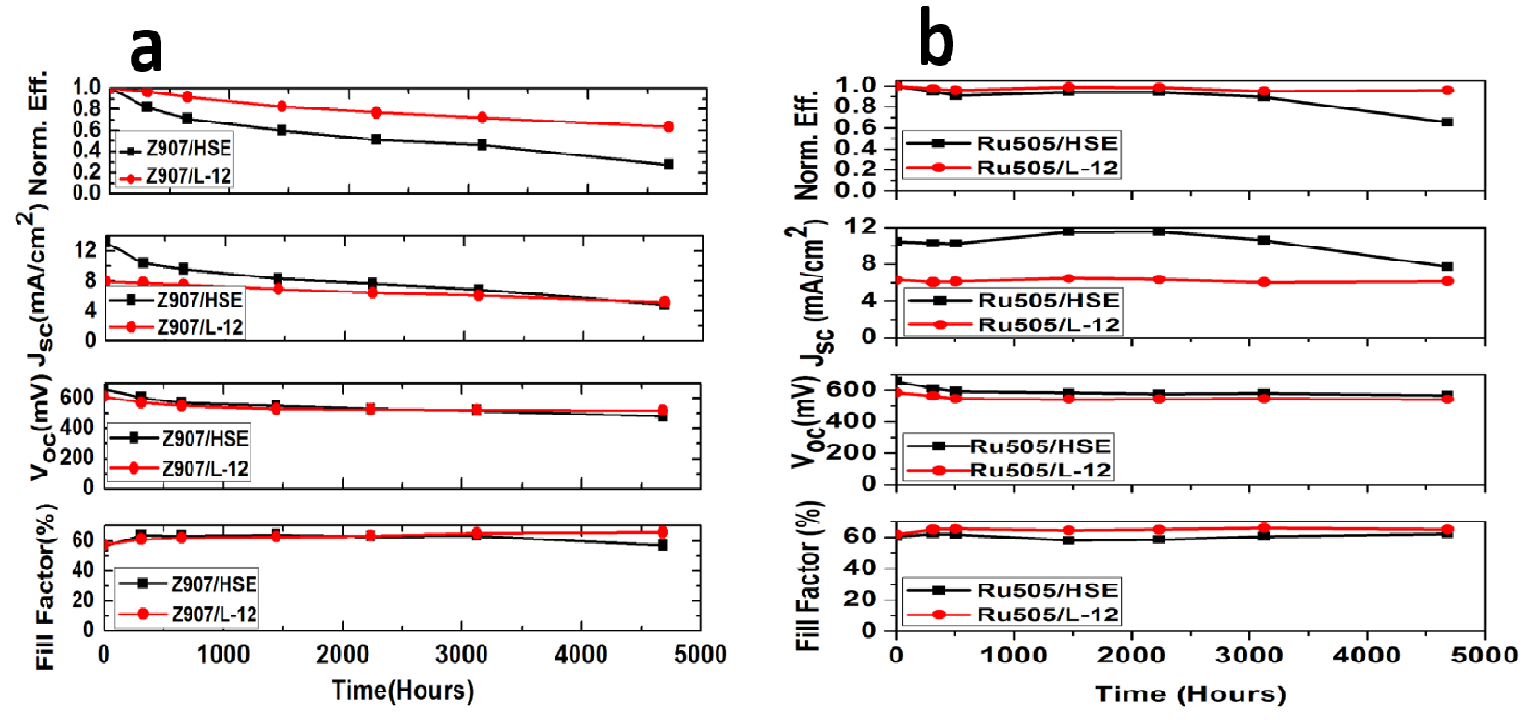

discussed by C. Han and S. Park, [16] the stress induced by the electrolyte expansion will break the encapsulation and permits the electrolyte leakage with the formation of island-type defects. Better encapsulation should solve this problem such as glass-frit [17].

Figure 2. The electrical parameters for a) Ru505/HSE and Ru505/L-12, b) Z907/HSE and Z907/L-12 under temperature stress at $85^{\circ} \mathrm{C}$ as a function of time under 1 sun AM $1.5 \mathrm{G} 1000$ $\mathrm{W} / \mathrm{m}^{2}$.

We used IS to analyze in depth the electrical differences associated to these two electrolytes,. Impedance spectra of Z907 devices is shown in Fig. 1(b). Similar behavior is found for Ru505 devices. Nyquist plots of Z907/HSE and Z907/L-12 fresh cells are shown in Fig.1(b). The features of this diagram are typically three semicircles in order of decreasing frequency from origin towards increasing real part of impedance at x-axis. The first arc at high frequency is describing the counter-electrode charge transfer resistance $R_{P t}$, the second one to describe the recombination resistance at the $\mathrm{TiO}_{2}$ /electrolyte interface $\mathrm{R}_{r e c}$ and finally the low-frequency arc represents the impedance of diffusion in the electrolyte and the width of each of these arcs corresponds to $R_{P t}, R_{r e c}$ and $\mathrm{R}_{\mathrm{d}}$, respectively, while the initial displacement of the arcs from the origin corresponds to the contribution from the FTO resistance $R_{S}[18,19]$. We use the fitting circuit shown in Fig. 3, where $\mathrm{C}_{\mathrm{pt}}$ is the capacitance at Pt electrode, $\mathrm{C}_{\mu}\left(=\mathrm{c}_{\mu} \cdot \mathrm{L}\right.$, where $\mathrm{L}$ is the length of the film) the chemical capacitance of the $\mathrm{TiO}_{2}$. $[18,20,21,22] R_{\text {series }}$ is the total series resistance given by 


$$
R_{\text {series }}=R_{s}+R_{d}+R_{P t}
$$

From these values, the total potential drop through the cell is calculated to obtain the potential at the semiconductor, VF. The electron diffusion length can be expressed in terms of the resistances as $L_{n}=L .\left(R_{r e d} / R_{t r}\right)^{1 / 2}$, and $R_{t r}\left(=r_{t r} \cdot L\right)$ is the transport resistance at corresponding applied potential [23]. The two more relevant changes observed in the spectra obtained for HSE and L12, see Fig. 1(b), are (i) the much larger diffusion resistance $\left(R_{d}\right)$ in L-12 electrolyte (larger arc at low frequency) and (ii) the displacement of the arcs towards the right associated to an increase in high frequency resistance $\left(R_{s}\right)$ of the device. The large value found for $R_{d}$ in L-12 electrolyte, which is one order of magnitude larger than for HSE electrolyte, was associated to the higher viscosity of L-12 with respect to HSE. [24] This fact produces mass transport limitations of iodide ions as described by [5, 25-27]

$$
K_{e t} \propto \vartheta^{-1}
$$

with $K_{e t}$ the charge transport rate constant in the electrolyte and $\vartheta$ its viscosity. The increase in $R_{s}$ is also attributed to this same effect: $R_{s}$ has contributions from the of TCO, wires and electrolyte bulk resistances. The increase observed is associated to this last contribution which also increases with viscosity. Note that the electrolyte contributes to resistance in two ways, the diffusion resistance in the diffusion layer (where there is a gradient of ion concentration) and the bulk resistance of electrolyte (where ion concentration is constant), which according to Hauch, is about half of electrolyte layer. [28] The large increase in $R_{d}$ is very likely the reason why short circuit current is so limited in L-12 samples and slow dye regeneration due to diffusion limitation

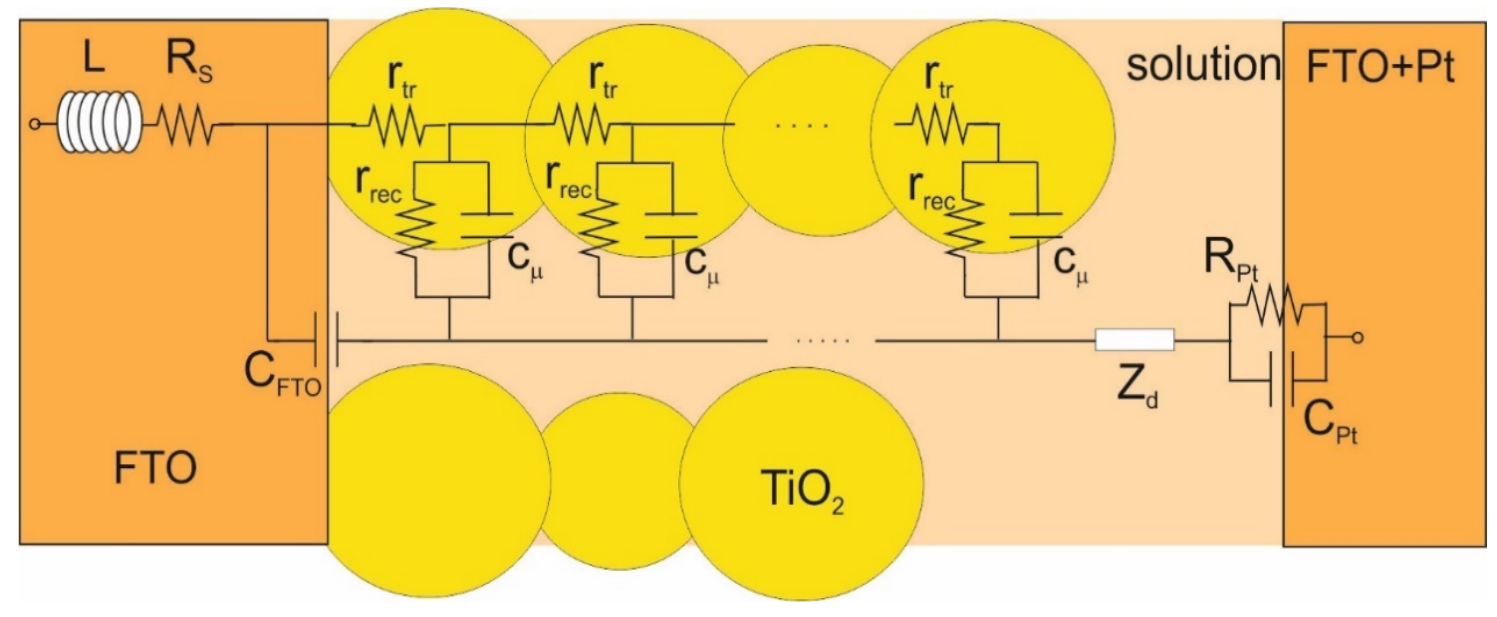

will

Figure 3 The transmission line model for impedance data fitting.

limit the ability of the dye for current production. Impedance spectra in Fig. 1(b) also show that for fresh cells changes in recombination at the $\mathrm{TiO}_{2} /$ dye/electrolyte interface and charge transfer at $\mathrm{Pt} / \mathrm{electrolyte}$ counter electrode are very small. In order to clarify the degradation mechanisms, 
strongly suffered DSCs employing Z907 sensitizer, IS data analysis of fresh samples in Fig. 1(b) was completed with the study of IS data of aged samples.

a

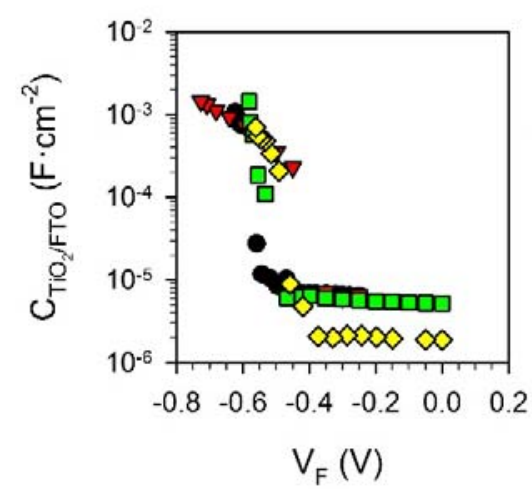

d

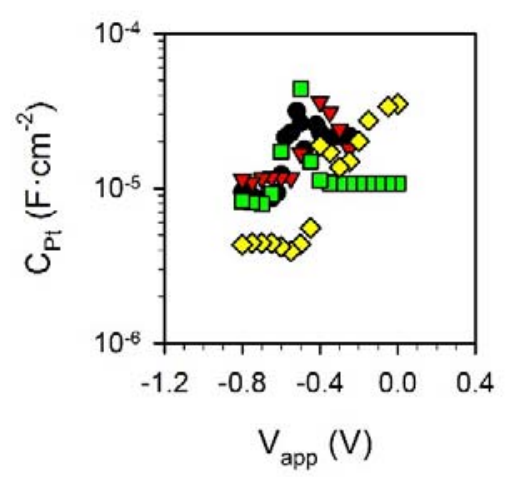

b

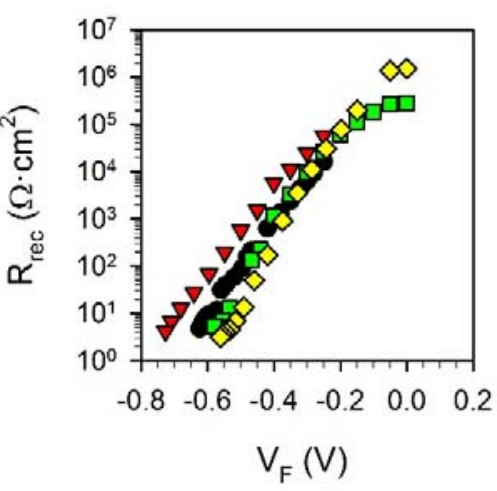

- Fresh L-12

$\nabla$ Fresh HSE

ㅁ Aged L-12

$\diamond \quad$ Aged HSE

e

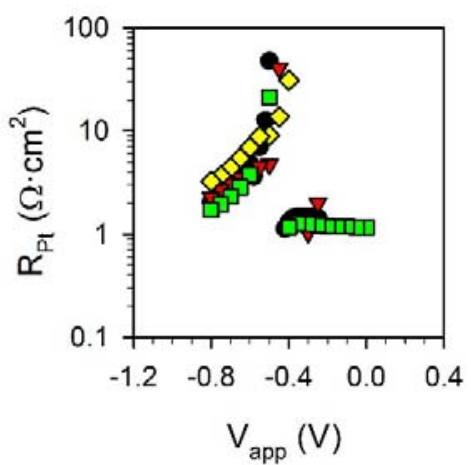

C

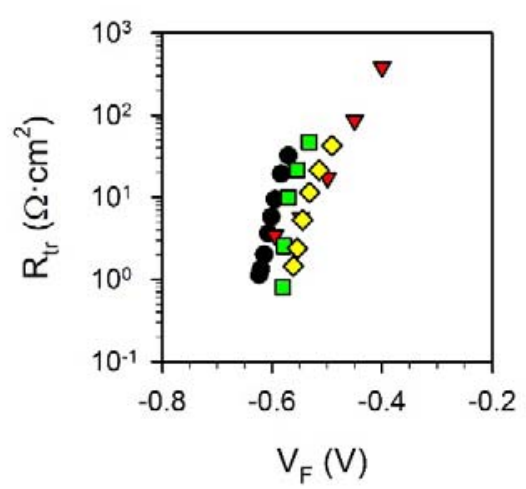

f

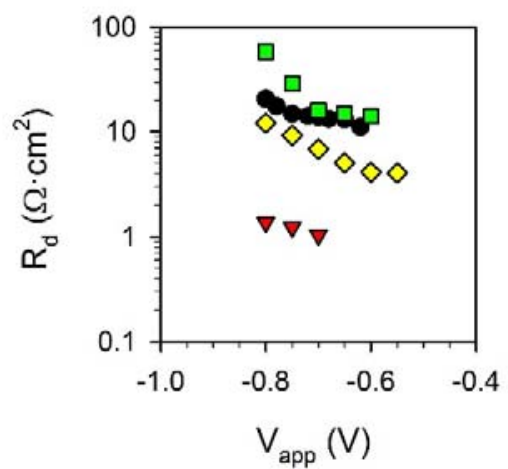

Figure 4. Evolution of (a) Bulk capacitance (b) recombination resistance of $\mathrm{TiO}_{2}$ electrode (c) transport resistance for electrons in the conduction band of $\mathrm{TiO}_{2}$ (d) Platinum counter electrode capacitance (e) Counter electrode charge transfer resistance and (f) resistance to diffusion of ions in the electrolyte respectively with applied voltage for fresh and aged cells.

Initially, at low applied voltages, the Fermi level is not high enough to inject electrons in the conduction band of $\mathrm{TiO}_{2}$ which makes the transport resistance very high at these potentials as seen in Fig. 4(c). Hence, at these potentials, we observe charging of the FTO/electrolyte interface. At moderate negative potentials, injection of electrons into the conduction band occurs, increasing the conductivity of $\mathrm{TiO}_{2}$, thus reducing the transport resistance. From this potential onwards, we observe a sharp increase in the capacitance which is associated with the chemical 
capacitance of $\mathrm{TiO}_{2}$. From Fig. 4(a) and Fig. 4(c), it appears that no significant change in the position of the conduction band occurs despite a change in the slope of the chemical capacitance and transport resistance for the Z907/HSE samples after aging. Variations in the slope of the capacitance versus voltage indicate a change in the density of mid-gap states. The aged Z907/HSE cells also shows lower resistance to recombination by almost one order fall at high negative potentials compared to fresh Z907/HSE cells. This is reflected directly in the shift of the exponential current density onset in the $J-V$ curve (Fig. 5) for aged Z907/HSE cells compared to fresh Z907/HSE cells. It is likely that the increased recombination kinetics is related to the formation of the $\left[\mathrm{D}^{+} \mathrm{I}_{3}^{-}\right]$complex in aged $\mathrm{Z907/HSE}$ cells, which can be seen in later section. The transport resistances after degradation appear not to be affected.

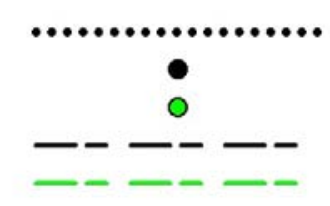

Fresh L-12 CV dark Fresh L-12 EIS dark Aged L-12 EIS dark Fresh L-12 CV light Aged L-12 CVlight

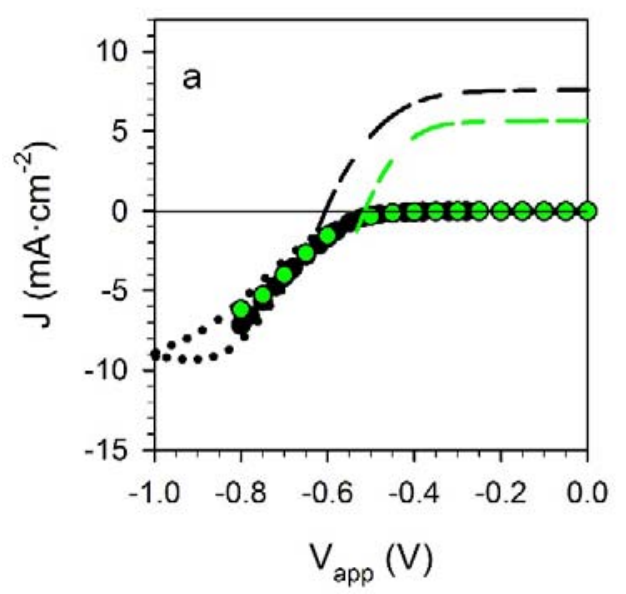

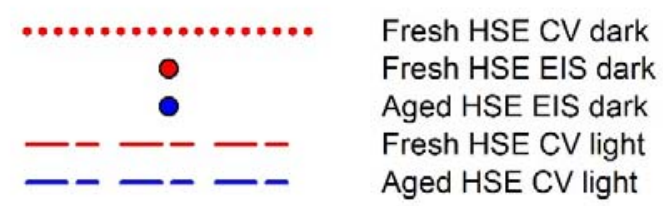

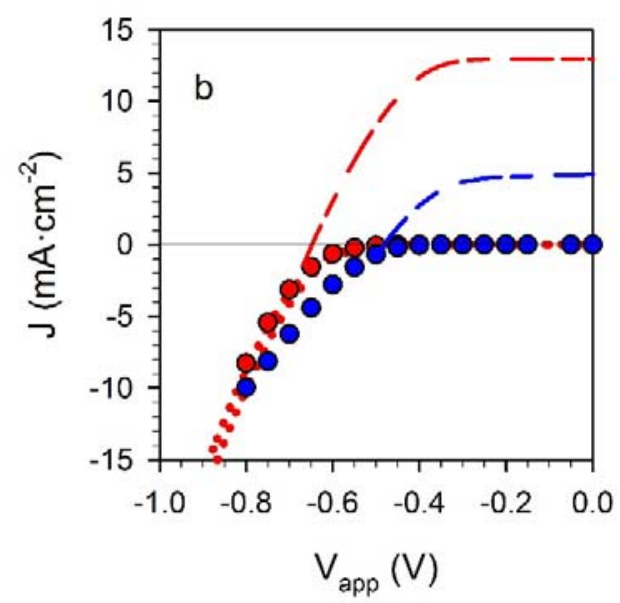

Figure $5 J-V$ curves obtained from IS and cyclic voltammetry (CV) measurements (a) Z907/L-12 cells (b) Z907/HSE cells.

We also note that the capacitance of the FTO/electrolyte interface in the case of the degraded sample has been reduced by half an order, see Fig. 4d. This may be related to the fact that electrolyte leakage associated to degradation reduces the FTO area in contact with electrolyte. On the contrary, capillarity still may preserve most of the electrolyte contact with the $\mathrm{TiO}_{2}$ surface.

Based on the shift observed in recombination resistance values of Fig. 4(b), a significant reduction in $V_{o c}$ of about $160 \mathrm{mV}$ in Z907/HSE cells may be expected for the aged Z907/HSE samples. In general, $V_{o c}$ decrease may occur due to a shift in the conduction band or changes in the kinetics of recombination [20]. Apparently, from Fig. 4(a) band (or capacitance) shift is small 
(even considering electrolyte leakage), therefore we can associate the drop in potential (and in $R_{\text {rec }}$ ) occurring in Z907/HSE sample to a large increase in recombination kinetics. The platinized counter electrode capacitance and corresponding resistance Figs. 4(d) and 4(e) respectively, appear to be fairly consistent for all the samples as expected, though the charge transfer resistance values show a peak at around $-0.5 \mathrm{~V}$. Again, decrease in $\mathrm{C}_{\mathrm{Pt}}$ for Z907/HSE sample may be associated to electrolyte leakage. In the case of Z907/L-12 samples, the recombination resistances of the fresh and aged samples shown in Fig. 4(b) show that the carrier recombination losses are only slightly accelerated by the aging process. The small decrease in recombination resistance is related to the $\sim 50 \mathrm{mV}$ shift towards lower potentials found in both transport resistances shown in Fig. 4(c) and capacitance in Fig. 4(a) of the aged sample. This shift indicates that the conduction band of the aged sample has been shifted to lower energies. As a consequence, from this data, for the aged Z907/L-12 samples, a slight decrease in $V_{o c}$, around $50 \mathrm{mV}$, is expected.

From $J-V$ curves under illumination shown in Fig. 5, we observe a reduction in $V_{o c}$ of $160 \mathrm{mV}$ for aged Z907/HSE samples compared to fresh ones, which matches the $V_{o c}$ shift predicted from IS measurements. We also observed a decrease in a large reduction in the $J_{s c}$, by $8.1 \mathrm{~mA} / \mathrm{cm}^{2}$ (Fig. 5) which was associated leakage. This electrolyte leakage is also responsible of the large increase of $R_{d}$ in aged HSE sample and thus to transport limitations as shown in Fig. 6. As observed in Fig. 4(f), the more viscous L-12 electrolyte presents a much larger diffusion resistance than HSE already in fresh cells. Consequently, maximum current attainable by L-12 cell is constrained by diffusion, see Fig. 6, while no limit is found for fresh HSE.

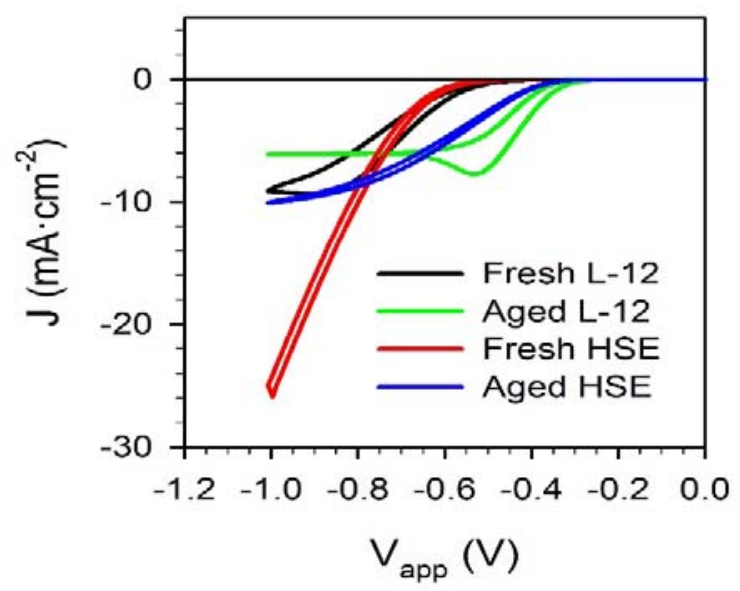

Figure 6 Dark $J-V$ curves of fresh and aged L-12 and HSE.

This implies that spacer between $\mathrm{TiO}_{2}$ film and counter electrode should be reduced to a minimum value to maximize $J_{s c}$ in L-12 samples. In aged samples, limiting current in L-12 decreases (and $R_{d}$ increases), producing an even stronger limitation to dye regeneration and 
consequently, a lower $J_{s c}$. This result may suggest $\mathrm{I}_{3}{ }^{-}$and iodide depletion upon aging. Finally, we observe a change in the slope of Rrec and the $J-V$ curves for aged cells in the dark and under illumination. The first may be related to the changes in the kinetics of recombination, while the second is also affected by the increase of $R_{\text {series }}$ (mainly $R_{d}$ ) found for these samples. Finally, for aged Z907/L-12 samples, from $J-V$ curves under illumination shown in Fig.5, we observe a $V_{o c}$ decrease of

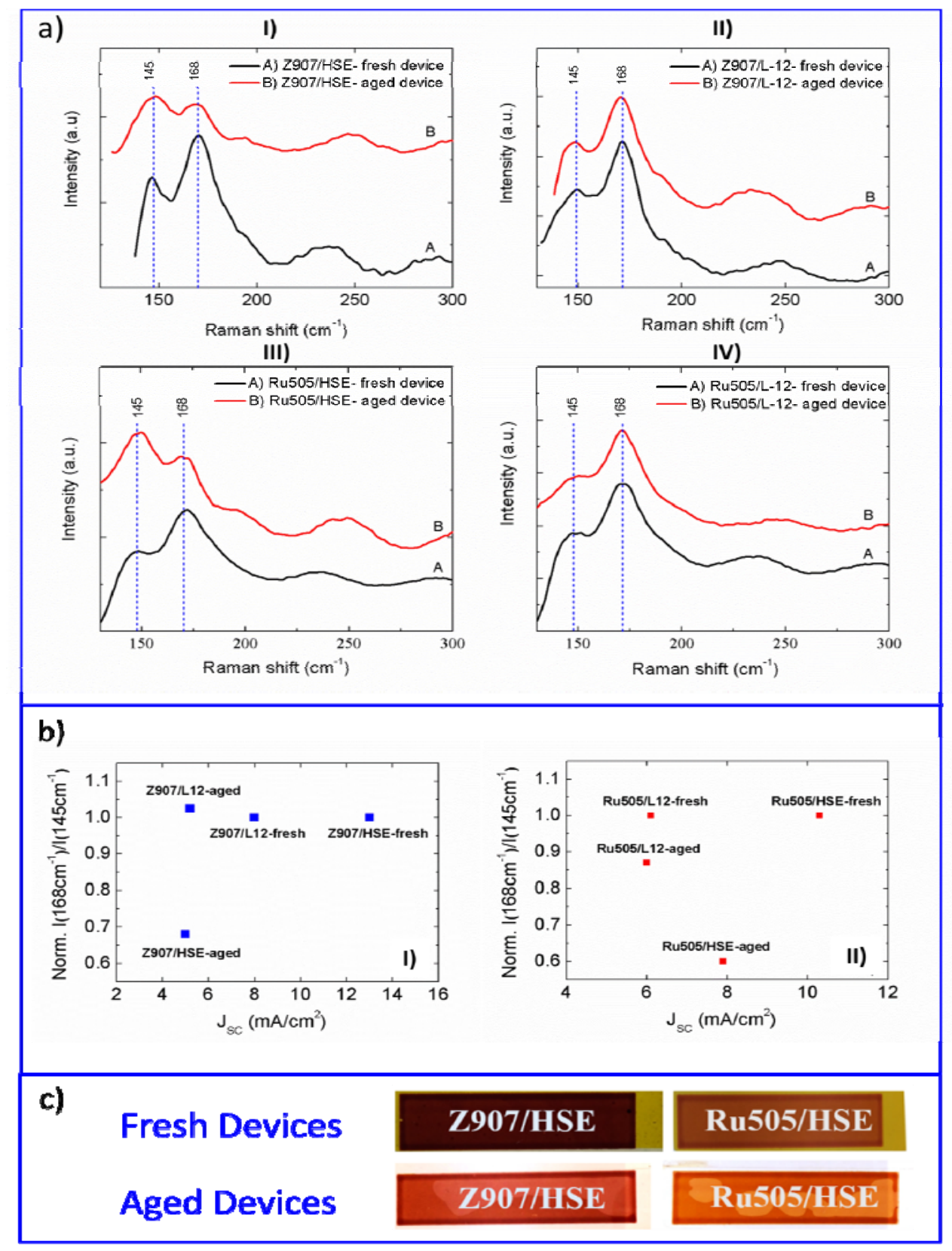

Figure 7 a) Low-frequency region of the Raman spectra acquired by focusing the laser beam $(514 \mathrm{~nm})$ onto the device's photo-electrode for fresh and thermal stressed cells (4700 hours at 
$85^{\circ} \mathrm{C}$ ). In particular, the different panels refer to I) Z907/HSE, II) Z907/L-12, III) Ru505/HSE, IV) Ru505/L-12. b) Intensity ratio for $\left(\mathrm{I}_{169} / \mathrm{I}_{145}\right)$ bands of all cells, collected from fresh and aged spectra of all the tested device typologies and normalizing these values by the numbers for corresponding fresh cells. c) Images of Z907/HSE and Ru505/HSE, fresh and aged DSCs after thermal stress at $85^{\circ} \mathrm{C}$.

$70 \mathrm{mV}$ for the aged sample, slightly higher than the predicted value from IS measurements in the dark. This difference may be associated to the decrease $\left(1.95 \mathrm{~mA} / \mathrm{cm}^{2}\right)$ in $J_{s c}$ observed for aged sample or an increase in the kinetics of recombination under illumination with aging.

Beside the breaking of the encapsulation induced by the thermal expansion of the electrolyte, several intrinsic factors cause a steady degradation of DSCs especially evident for the Z907 based cells (Fig. 2a). These factors are systematically investigated by means of Raman spectroscopy and impedance spectroscopy. Low-frequency region Micro-Raman spectra of fresh and aged devices are reported in Fig. 7(a), while complete Raman spectra related to fresh Z907 and Ru505 based photoelectrodes have been extensively discussed in literature [4, 29, 30]. In particular, all the reported Raman spectra exhibit the characteristic Raman-active phonon modes of the tetragonal structure of anatase $\mathrm{TiO}_{2}$ at $145 \mathrm{~cm}^{-1}$ [31-33] that will be employed as reference peak for the following description, since $\mathrm{TiO}_{2}$ mesoporous layer is known to be unaffected by prolonged thermal stress [34]. In all devices, an important interaction between excited dye ( $\left.\mathrm{D}^{+}\right)$ and tri-iodide $\left(\mathrm{I}_{3}{ }^{-}\right)$ions can be detected in Micro-Raman spectra at $169 \mathrm{~cm}^{-1}$ assigned to the $\mathrm{I}_{3}{ }^{-}$ symmetric vibration in the $\left[\mathrm{D}^{+}-\mathrm{I}_{3}^{-}\right]$complex.

Raman band of the $\left[\mathrm{D}^{+} \mathrm{I}_{3}{ }^{-}\right]$complex at $169 \mathrm{~cm}^{-1}$ probed via the photoelectrode side were found to be highly sensitive to the electrolyte composition variations induced by thermal stress [14]. To quantify this variation, the intensity ratio of the $169 \mathrm{~cm}^{-1}$ band relative to the most intense $\mathrm{TiO}_{2}$ band at $145 \mathrm{~cm}^{-1}\left(\mathrm{I}_{169} / \mathrm{I}_{145}\right)$ is extracted by the Raman spectra for each investigated device and reported in Fig. 7(b) with respect to the $J_{s c}$ recorded under 1 sun illumination. In fact, a clear correlation between the electrical cell parameters and the relative intensity of the $169 \mathrm{~cm}^{-1}$ mode was already evidenced by Kontos and coworkers, [14] reflecting the depletion of bound triiodides at the dye/ $\mathrm{TiO}_{2}$ interface. A dramatic decline in the intensity ratio was found for the thermal stressed cells Z907/HSE and Ru505/HSE by $\sim 32 \%$ and $\sim 40 \%$ respectively, that is consistent with the strong $J_{s c}$ decrease reported in Fig. 7(b) for both Z907/HSE and Ru505/HSE devices. The images of fresh and aged cell of Z907/HSE and Ru505/HSE respectively, shown in Fig. 7(c). The above mentioned reduction of $\mathrm{I}_{169} / \mathrm{I}_{145}$ ratio can be ascribed to the triiodide loss at the photo-electrode due to the breaking of the sealing in HSE and the following electrolyte leakage mentioned previously after prolonged annealing. As a consequence, the aged devices showed a dramatic reduction in efficiency independently from the employed dye $(70 \%$ for Z907/HSE and $40 \%$ for Ru505/HSE). On the contrary, thermal stressed Ru505/L-12 based devices showed a moderate decline of $\mathrm{I}_{169} / \mathrm{I}_{145}$ ratio (-13.3\%) with respect to their corresponding fresh samples while Z907/L-12 exhibited a slight increase $(+2.5 \%)$ of the considered peak intensity ratio. 
The $\left[\mathrm{D}^{+} \mathrm{I}_{3}^{-}\right]$complex increase can be imputed to the partial loss of coordinated $\mathrm{SCN}^{-}$ligands from the dye molecules [30], that retains dye in a prolonged oxidized state. Thse chages in $\mathrm{TiO} 2$ surface may be responsible of the small conduction band shift observed with impedance. In this context, the coordination between $\mathrm{D}^{+}$and triiodide ions is favored and the formation of $\mathrm{Ru}(\mathrm{III})$ intermediates finally leading to $\mathrm{Ru}(\mathrm{II})(\mathrm{CN})$ become a possible degradation-induced sub-product $[4,35,36]$. The interaction between dye and electrolyte accelerates the recombination reaction, that is associated to the decrease in recombination resistance for aged L-12 devices that slowsdown the device's dynamics reflected in a progressive photovoltaic performance reduction [37], as shown in Fig. 2a. Since the Raman spectrum of the electrolyte acquired on the electrolyte monitor window of thermal stressed Z907/L12 sample (reported in our previous publication [30] didn't show any Raman features of dye molecules, it is possible to assert that dye is still anchored on $\mathrm{TiO}_{2}$ surface after thermal stress by excluding desorption phenomena. Notably, the absence of any relevant and evident modification in low-frequency Raman spectrum of Ru505/L12 reported in Fig. 7(a)-IV confirms the chemical stability of the device's constituents and it supports the stable efficiency trend Fig. $2 b$ under prolonged thermal stress. In fact, devices employing Ru505 dye showed improved stability under thermal stress conditions due to the stronger coordination to ruthenium of $\mathrm{CN}^{-}$with respect to the $\mathrm{SCN}^{-}$ligand $[4,14]$. Thus, the use of a Ru-based dye with cyanide group as ancillary ligands coupled with a more viscous electrolyte (such as L-12 tested in this work) can effectively prevent dye/electrolyte interface degradation under prolonged annealing condition $\left(85^{\circ} \mathrm{C}\right)$.

\section{Conclusion}

The investigated DSC devices Z907/HSE, Z907/L-12, Ru505/HSE and Ru505/L-12 hàve shown different degradation rates because of different viscosity of electrolytes and thermal expansion coefficients under thermal aging. The shown data revealed the cells with viscous electrolyte (L12) are less efficient due to charge transport limitations and higher diffusion resistance but more stable than the cells employing HSE independently from the used dyes (Z907, Ru505). The most degraded device was Z907/HSE while Ru505/L-12 was the most stable one under thermal stress. As a result of thermal stress, there were conformational changes after 4700 hours of stress, devices configured with HSE electrolyte suffered harsh degradation because of thermal expansion of MPN based electrolyte, which generated enough pressure to break the sealing and cause electrolyte leakage. On the contrary, the devices with L-12 undergo moderate degradation under thermal stress, mainly because the ionic liquid based electrolyte is not able to generate enough pressure to break the sealing at $85^{\circ} \mathrm{C}$. The most prominent effect of thermal aging in the Raman spectra is the reduction in the intensity ratio of the $\mathrm{I}_{169} / \mathrm{I}_{145}$ ascribed to the interaction between the dye and triiodide species, which could be a possible source of increased recombination kinetics. On the other hand, the $\mathrm{I}_{169} / \mathrm{I}_{145}$ ratio variation upon thermal aging for devices employing L-12 electrolyte is negligible by confirming the chemical stability of dye/electrolyte interface. The significant reduction in $V_{o c}$ of about $160 \mathrm{mV}$ is mainly attributed to electrolyte leackage that produced enhanced recombination kinetics in Z907/HSE cells. L-12 
cells only shows small changes in recombination kinetics, which were associated to interaction of triiodide species with the excited state of dye described by RAMAN. This fact coupled with the ower rate of thermal expansion as compared to HSE cells, provide excellent stability to devices under thermal aging. Finding the best sealing for such class of devices for long term use in plausible operating conditions is still an open issue. Therefore, our study reveals that hermetic sealing and device configuration such as Ru505/L-12 have the long term stable DSCs and future for marketable technology.

\section{Supporting Information}

(J-V) curves for fresh cells Ru505/HSE and Ru505/L-12 solar cells under 1 sun illumination. (Figure S1). The UV-vis spectra and chemical structure of Z907 dye and Ru505 dye (Figure S2).

\section{Corresponding Author}

Phone : +390672597456; Fax : +39-06-72597939, E-mail : aldo.dicarlo@uniroma2.it.

\section{Notes}

The authors declare no competing financial interest.

Acknowledgments: The research leading to these results has received funding from the European Union Seventh Framework Program [FP7/2007- 2013] under Grant Agreement 316494. S.R. acknowledges Generalitat Valenciana for the grant GRISOLIA/2014/034.

\section{References}

1. O'Regan, B.; Grätzel, M. A low-cost, high-efficiency solar cell based on dye-sensitized colloidal $\mathrm{TiO}_{2}$ films, Nature 1991, 353,737-739.

2. Osterwald, C. R.; McMahon, T. J. History of Accelerated and Qualification Testing of Terrestrial Photovoltaic Modules: A Literature Review, Prog. Photovolt: Res. Appl. 2009, 17,11-33.

3. Figgemeier, E.; Hagfeldt, A. Are dye-sensitized nano-structured solar cells stable? An overview of device testing and component analyses, Int. J. Photoenergy 2004, 6, 127-140.

4. Agresti, A.; Pescetelli, S.; Quatela, A.; Mastroianni, S.; Brown, T.M.; Reale, A.; Bignozzi, C.A.; Caramori, S.; di Carlo, A. Micro-Raman analysis of reverse bias stressed dye-sensitized solar cells, RSC Adv. 2014, 4, 12366-12375.

5. Gorlov, M.; Kloo, L. Ionic liquid electrolyte for dye-sensitized solar cells, Dalton Tran. 2008, 2655-2666.

6. Wheatley, M.G.; McDonagh, A.M.; Brungs, M.P.; Chaplin, R.P.; Sizgek, E. A study of reverse bias in a dye sensitized photoelectrochemical device, Solar Energy Materials \& Solar Cells 2003, 76, 175-181.

7. Lin Ke, Dolmanan, S. B.; Shen, L.; Pallathadk, P. K.; Zhang, Z.; Lai, D. M. Y.; Liu, H. Degradation mechanism of ZnO-based dye-sensitized solar cells, Solar Energy Materials \& Solar Cells 2010, 94, 323-326. 
8. Toivola, M.; Halme, J.; Peltokorpi, L.; Lund, P. Investigation of temperature and aging effects in nanostructured dye solar cells studied by electrochemical impedance spectroscopy, International Journal of Photoenergy 2009, 786429, 15.

9. Tanaka, H.; Takeichi, A.; Higuchi, K.; Motohiro, T.; Takata, M.; Hirota, N.; Nakajima, J.; Toyoda, T. Long-term durability and degradation mechanism of dye-sensitized solar cells sensitized with indoline dyes, Solar Energy Materials \& Solar Cells 2009, 93, $1143-$ 1148 .

10. Mathew, A.; Rao, G. M.; Munichandraiah, N. Towards fabrication of stable dye sensitized solar cells based on acetonitrile as solvent for the redox couple, Adv. Mat. Lett. 2014, 5(4), 180-183.

11. Harikisun, R.; Desilvestro, H. Long-term stability of dye solar cells, Solar Energy 2011, $85,1179-1188$.

12. Stergiopoulos, T.; Kontos, A. G.; Jiang, N.; Milliken, D.; Desilvestro, H.; Likodimos, V.; Falaras, P. High boiling point solvent-based dye solar cells pass a harsh thermal ageing test, Solar Energy Materials \& Solar Cells, 2016,144, 457-466.

13. Jiang, N.; Sumitomo, T.; Lee, T.; Pellaroque, A.; Bellon, O.; Milliken, D.; Desilvestro, H. High temperature stability of dye solar cells, Solar Energy Materials \& Solar Cells, 2013, $119,36-50$.

14. Kontos, A. G.; Stergiopoulos, T.; Likodimos, V.; Milliken, D.; Milliken, D.; Tulloch, G.; Falaras, P. Long-Term Thermal Stability of Liquid Dye Solar Cells, J. Phys. Chem. C, 2013, 117, 8636-8646.

15. Yang, W.; Hao, Y.; Ghamgosar, P.; Boschloo, G. Thermal Stability Study of DyeSensitized Solar Cells with Cobalt Bipyridyl-based Electrolytes, Electrochimica Acta, 2016, 213, 879-886.

16. Han, C.; Park, S. Development and Validation of Dye-sensitized Solar Cell Finite Element Model for Sealing Failure Investigation, 15th International Conference on Thermal, Mechanical and Multi-Physics Simulation and Experiments in Microelectronics and Microsystems, Euro Sim E, 2014.

17. Sastrawan, R.; Beier, J.; Belledin, U.; Hemming, S.; Hinsch, A.; Kern, R.; Vetter, C.; Petrat, F.M.; Prodi-Schwab, A.; Lechner, P.; Hoffmann, W.; A glass frit-sealed dye solar cell module with integrated series connections, Solar Energy Materials and Solar Cells 2006, 90, 11, 1680-1691.

18. Fabregat-Santiago, F.; Bisquert, J.; Garcia-Belmonte, G.; Boschloo, G.; Hagfeldt, A. Influence of electrolyte in transport and recombination in dye-sensitized solar cells studied by impedance spectroscopy, Solar Energy Materials \& Solar Cells, 2005, 87, 117-131.

19. Fabregat-Santiago, F.; Bisquert, J.; Garcia-Belmonte, Mora-Sero, I.; Bisquert, J. Characterization of nanostructured hybrid and organic solar cells by impedance spectroscopy, Phys. Chem. Chem. Phys., 2011, 13, 9083-9118.

20. Agrell, H. G.; Lindgrenb, J.; Hagfeldt, A. Coordinative interactions in a dye-sensitized solar cell, Journal of Photochemistry and Photobiology A: Chemistry, 2004, 164,23-27.

21. Raga, S. R.; Barea, E. M.; Fabregat-Santiago, F. Analysis of the Origin of Open Circuit Voltage in Dye Solar Cells, J. Phys. Chem. Lett., 2012, 3, 1629-1634.

22. Halme, J.; Vaherma, P.; Miettunen, K.; Lund, P. Device physics of dye solar cells, Adv. Mater., 2010, 22, 210-234. 
23. Mastroianni, S.; Lanuti, A.; Penna, S.; Reale, A.; Brown, T. M.; Di Carlo, A.; Decker, F. Physical and Electrochemical Analysis of an Indoor-Outdoor Ageing Test of Large-Area Dye Solar Cell Devices, Chem. Phys. Chem., 2012, 13, 2925-2936.

24. Fabregat-Santiago, F.; Bisquert, J.; Palomares, E.; Otero, L.; Kuang, D.; Zakeeruddin, S. M.; Grätzel, M. Correlation between photovoltaic performance and impedance spectroscopy of dye-sensitized solar cells Based on Ionic Liquids, J. Phys. Chem. C, 2007, 111, 6550-6560.

25. Bisquert, J. Theory of the Impedance of Electron Diffusion and Recombination in a Thin Layer, J. Phys. Chem. B, 2002, 106, 325-333.

26. Zusman, L.D. Dynamical solvent effect in electron transfer reaction, Int. j. of res. in phys. chem. and chem. Phy. 1994, 186, 1-29.

27. Toivola, M.; Ahlskog, F.; lund, P. Industrial sheet metal for nanocrystaline dye sensitized solar cell structures, Solar Energy Materials \& Solar Cells, 2006, 90, 28812893.

28. Hauch, A.; Georg, A. Diffusion in the electrolyte and charge-transfer reaction at the platinum electrode in dye-sensitized solar cells, Electrochimica Acta, 2001, 46, 34573466.

29. Likodimos, V.; Stergiopoulos, T.; Falaras, P.; Harikisun, R.; Desilvestro, J.; Tulloch, G. Prolonged light and thermal stress effects on industrial dye-sensitized solar cells: a microraman investigation on the long-term stability of aged cells, J. Phys. Chem. C, 2009, 113, 9412-9422.

30. Sarker, S.; Seo, H. W.; Kim, D. M. Electrochemical impedance spectroscopy of dyesensitized solar cells with thermally degraded N719 loaded $\mathrm{TiO}_{2}$, Chem. Phys. Lett., 2013, 585 193-197.

31. Ohsaka, T.; Izumi, F.; Fujiki J. Y. Raman spectrum of anatase, $\mathrm{TiO}_{2}$, Raman Spectrosc., 1978,7, 321-324.

32. Duffy, N. W.; Dobson, K. D.; Gordon, K. C.; Robinson, B. H.; McQuillan, A. J. In situ infrared spectroscopic analysis of the adsorption of ruthenium(II) bipyridyl dicarboxylic acid photosensitisers to $\mathrm{TiO}_{2}$ in aqueous solutions, Chem. Phys. Lett., 1997, 266, 451455.

33. Perez Leon, C.; Kador, L.; Peng, B.; Thelakkat, M. Characterization of the adsorption of Ru-bpy Dyes on mesoporous $\mathrm{TiO}_{2}$ films with UV-Vis, Raman, and FTIR spectroscopies, J. Phys. Chem. B, 2006, 110, 8723-8730.

34. Likodimos, V.; Stergiopoulos, T.; Falaras, P.; Harikisun, R.; Desilvestro, J.; Tulloch, G. Prolonged light and thermal stress effects on industrial dye-sensitized solar cells: a microraman investigation on the long-term stability of aged cells, J. Phys. Chem. C, 2009, 113, 9412-9422.

35. Svensson, P. H.; Kloo, L. Synthesis, structure, and bonding in polyiodide and metal iodide-iodine systems, Chem. Rev., 2003, 103, 5, 1649-1684.

36. Agresti, A.; Pescetelli, S.; Gatto, E.; Venanzi, M.; Di Carlo, A. Polyiodides formation in solvent based Dye Sensitized Solar Cells under reverse bias stress, Journal of Power Sources, 2015, 287, 87-95.

37. O’Regan, B.; Lopez-Duarte, I.; Martinez-Diaz, M. V.; Forneli, A.; Albero, J.; Morandeira, A.; Palomares, E.; Torres, T.; Durrant, J. R. Catalysis of recombination and its limitation on open circuit voltage for dye sensitized photovoltaic cells using phthalocyanine dyes, J. Am. Chem. Soc., 2008, 130, 2906-2907. 


\title{
Table of Content Image
}

\author{
Graphical Abstract
}

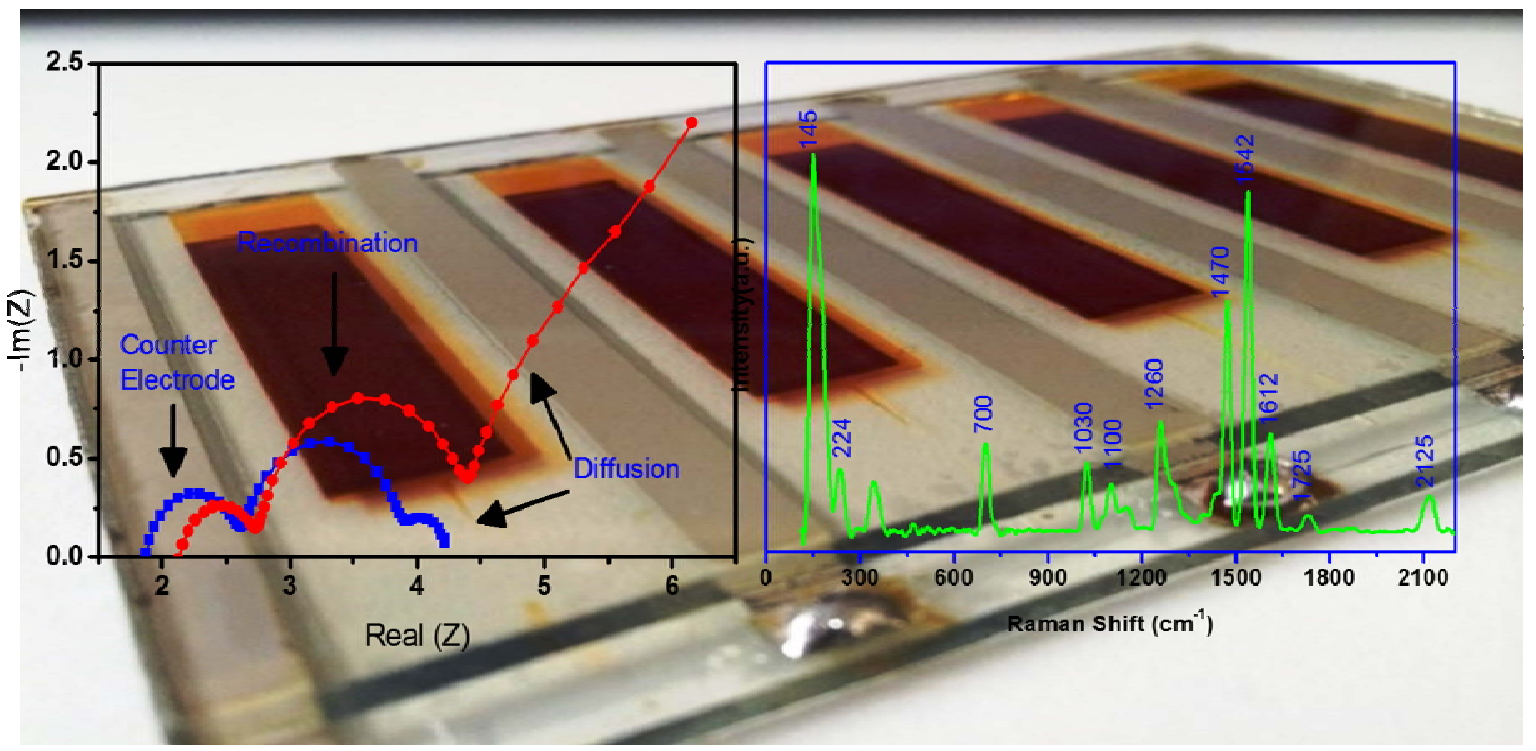

The graphical abstract contains actual master plate image consist of 5 individual equivalent cells. The stability study reveal the degradation mechanism with the help of Electrochemical Impedance Spectroscopy (Left side panel) and micro Raman Spectroscopy (Right side panel). 